\title{
Radial Electron Temperature and Density Measurements Using Thomson Scattering System in GAMMA 10/PDX
}

\author{
M. Yoshikawa ${ }^{a^{*}}$, K. Ohta ${ }^{a}$, X. Wang ${ }^{a}$, M. Chikatsu ${ }^{a}$, J. Kohagura ${ }^{a}$, Y. Shima ${ }^{a}$, \\ M. Sakamoto ${ }^{a}$, T. Imai ${ }^{a}$, Y. Nakashima ${ }^{a}$, R. Yasuhara ${ }^{b}$, I. Yamada ${ }^{b}$, H. Funaba ${ }^{b}$, and \\ T. Minami ${ }^{c}$ \\ ${ }^{a}$ Plasma Research Center, University of Tsukuba, \\ Tsukuba, Ibaraki 305-8577, Japan \\ ${ }^{b}$ National Institute for Fusion Science, \\ Toki, Gifu 509-5292, Japan \\ ${ }^{c}$ Institute of Advanced Energy, Kyoto University, \\ Uji, Kyoto 611-0011, Japan \\ E-mail: yosikawa@prc.tsukuba.ac.jp
}

\begin{abstract}
A Thomson scattering (TS) system in GAMMA 10/PDX has been developed for the measurement of radial profiles of electron temperature and density in a single plasma and laser shot. The TS system has a large solid angle optical collection system and high-sensitivity signal detection system. The TS signals are obtained using four-channel high-speed digital oscilloscopes controlled by a Windows PC. We designed the acquisition program for six oscilloscopes to obtain $10-\mathrm{Hz}$ TS signals in a single plasma shot, following which the time-dependent electron temperatures and densities can be determined. Moreover, in order to obtain larger TS signal intensity in the edge region, we added a second collection mirror. The radial electron temperatures and densities at six radial positions in GAMMA 10/PDX were successfully obtained.
\end{abstract}

KEYWORDS: Plasma diagnostics - Thomson scattering; GAMMA 10/PDX; electron temperature 


\section{Contents}

1. Introduction

2. Thomson scattering system in GAMMA 10/PDX 1

3. Additional mirror effects 3

4. Radial electron temperature and density measurements 4

5. Summary 5

\section{Introduction}

Thomson scattering (TS) diagnostic is one of the most useful methods for measuring electron temperature and density of plasmas [1-10]. TS systems are normally applied to plasma densities of over $10^{19} \mathrm{~m}^{-3}$. In lower-density plasmas, such as peripheral plasmas or divertor plasmas, an effective TS system for signal enhancement is needed. TS signal depends linearly on the incident laser power, electron density, and solid angle of TS collection optics. In GAMMA 10/PDX, a TS system with a large solid angle optical collection system has been developed [810]. GAMMA 10/PDX is an effectively axisymmetrized minimum-B anchored tandem mirror having thermal barrier at both end-mirrors and an end divertor experimental module in the end cell [11-12]. The $\mathrm{x}$-axis and $\mathrm{y}$-axis are perpendicular to the magnetic field in the horizontal and vertical directions, respectively. The z-axis is parallel to the magnetic field. The plasma is created by plasma guns, and heated and sustained using ion cyclotron heating (ICH) systems. There are three types of oscillators, called RF1, RF2, and RF3. The waves excited by the RF1 (9.9-10.3 MHz) and RF2 ( 6.36 MHz) systems handle plasma production and ion heating in the central cell, respectively. The RF3 $(\sim 10.3 \mathrm{MHz})$ system is used to increase the electron density. In GAMMA 10/PDX, the electron temperature, electron density, and ion temperature are measured by the TS system, a 70-GHz interferometer system, and charge-exchange neutral particle analyzer, respectively. The electron density, electron temperature, and ion temperature are typically about $40 \mathrm{eV}, 2 \times 10^{18} \mathrm{~m}^{-3}$, and $5 \mathrm{keV}$, respectively. In former experiments, we could measure five radial positions in a single laser shot in a single plasma shot [10]. Then we improved the TS system for six-position measurements in a single laser shot in a single plasma shot, adding multi-channel optical fibers, a polychromator, and a high-speed oscilloscope. Moreover, we included an additional mirror to the optical collection system for enhancing the TS signals in the edge plasma region. We designed a multi-time data acquisition program for oscilloscopes operating at $10 \mathrm{~Hz}$.

In this paper, we describe the improved TS system and the results of radial electron temperature and density measurements in GAMMA 10/PDX. The additional mirror set over the main collection mirror improves the TS signal intensity. We applied the TS system to hot-ionmode plasma to obtain time-dependent electron temperature and density.

\section{Thomson scattering system in GAMMA $10 / P D X$}


The TS system is installed in the central cell of GAMMA 10/PDX. A schematic of the TS system is shown in Fig. 1. This system is a modification of the former TS system $[13,14]$. A horizontally polarized laser beam from the yttrium-aluminum-garnet (YAG) laser (Continuum, Powerlite 9010, $2 \mathrm{~J} /$ pulse, pulse width of $10 \mathrm{~ns}, 10 \mathrm{~Hz}$, and beam diameter of about $9 \mathrm{~mm}$ ) passes through mirrors and irises before being focused onto the plasma center by the first convex lens (Shigmakoki, $f=2000 \mathrm{~mm}, \phi=50 \mathrm{~mm}$ ) at the downside port window. After interacting with the plasma, the laser beam is emitted from the upper-side port window. A mirror flipper and beam dump for the single pass system are installed. In the normal single-pass TS configuration, the mirror flipper is closed and the laser beam is dumped by the beam dump. We used an $\mathrm{Al}: \mathrm{SiO}_{2}$-coated spherical mirror with a curvature radius of $1.2 \mathrm{~m}$ and a diameter of $0.6 \mathrm{~m}$ for the TS light-collection optics. The $90^{\circ}$-scattered light is collected and reflected by the spherical mirror, after which it reaches 9-channel optical fiber bundles with a cross section of 2 $\times 7 \mathrm{~mm}^{2}$. The 6.67 -m-long optical fiber bundles are connected to six 5-channel polychromators. The TS system can measure the radial positions of $X=0, \pm 5, \pm 10, \pm 15, \pm 20 \mathrm{~cm}$ by selecting the appropriate optical fibers. The polychromator is composed of five relay and collection lenses, five interference filters, and five silicon avalanche photodiodes (PerkinElmer, C30659-1060$3 \mathrm{AH}$, bandwidth of $50 \mathrm{MHz}$ ) with additional preamplifiers (Tokyo Opto-Electronics, PLM12A001-2) for increasing the output signal intensities. Measured wavelengths of the polychromator are $1059 \pm 2 \mathrm{~nm}(\mathrm{CH} .1), 1055 \pm 2 \mathrm{~nm}$ (CH. 2), $1050 \pm 3 \mathrm{~nm}$ (CH. 3), $1040 \pm 7$ $\mathrm{nm}(\mathrm{CH} .4)$, and $1020 \pm 14 \mathrm{~nm}(\mathrm{CH} .5)$. A four-channel high-speed oscilloscope (IWATSU, DS5524A) is used to measure four wavelength channels with a bandwidth of $200 \mathrm{MHz}$ and a sampling rate of $1.0 \mathrm{GS} / \mathrm{s}$. The measured signals are recorded by a Windows PC using the IWATSU multi-oscilloscope control software (IWATSU, MultiVControl V2.23). The electron temperatures are obtained using the chi-square method.

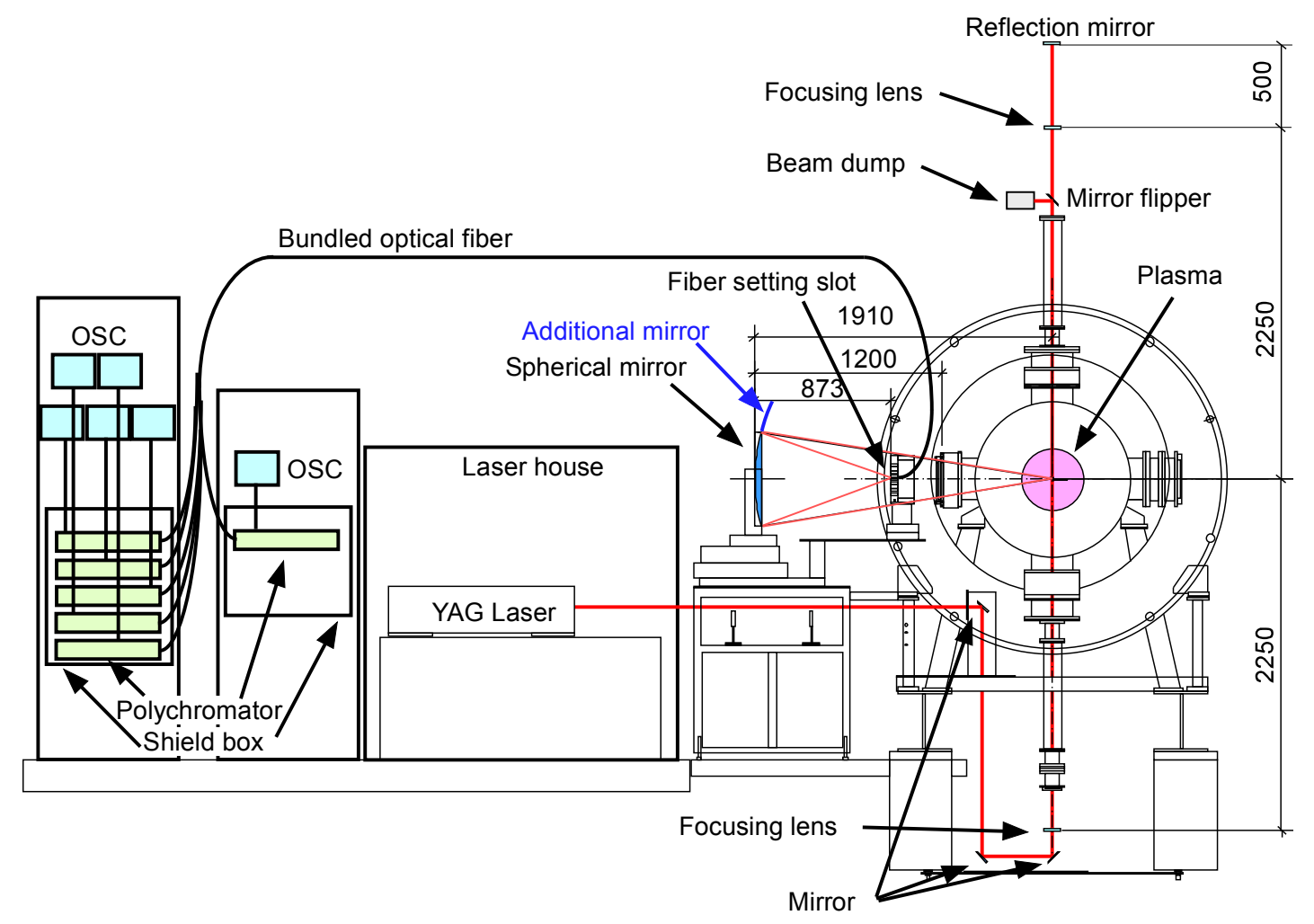

Figure 1. The TS system in GAMMA 10/PDX. 
In the edge plasma region of about $|\mathrm{X}|>15$ $\mathrm{cm}$, the TS signal is much lower than that in the center region. The TS collection optics show that the relative intensities at $|X|=10,15$, and $20 \mathrm{~cm}$ are about $0.7,0.5$, and 0.2 times that at the plasma center, respectively. Then, we included an additional mirror for improving the TS light intensity. $\mathrm{An} \mathrm{Al}: \mathrm{SiO}_{2}-$ coated spherical mirror with a curvature radius of $1.2 \mathrm{~m}$ and diameter of $0.2 \mathrm{~m}$ is used. It is set on top of the main collection mirror. The distance between the main mirror center and the additional mirror center is about $0.44 \mathrm{~m}$. The mirror size is optimal for obtaining the maximum collection efficiency, considering the solid angle and setting position. With the additional mirror, the TS signal intensities at $\mathrm{X}$ $=-10 \mathrm{~cm},-15 \mathrm{~cm}$, and $-20 \mathrm{~cm}$ are about $1.2,1.4$, and 2.6 times those without it, respectively, as determined from the calculation using the optical design software ZEMAX and solid angle calculation.

\section{Additional mirror effects}

Rayleigh and Raman gas scattering experiments were carried out for setting up the optical system and evaluation of the TS system. Nitrogen gas is used, and the pressure in the GAMMA 10/PDX device is increased to 200 Torr. The output Raman scattering signals of all channels of the polychromator are added to obtain the integrated intensities. In Fig. 2 (a), (b), and (c), the integrated Raman scattering intensities with and without additional mirror against nitrogen gas pressures at $\mathrm{X}=-10 \mathrm{~cm}$, $-15 \mathrm{~cm}$, and $-20 \mathrm{~cm}$, respectively, are shown. With the additional mirror, the scattering signal intensity increases to about $1.2 \pm 0.3,1.5 \pm 0.2$ and $3.1 \pm 0.6$ times those without the mirror, respectively, which match the calculation results mentioned above.

With the application of the TS system to the GAMMA 10/PDX plasma, the TS scattered signal intensities are increased when using the additional mirror. In Fig. 3 (a) and (b), the TS signals at $X=-10 \mathrm{~cm}$ and $-15 \mathrm{~cm}$, respectively, are shown. Unfortunately, at $\mathrm{X}=$
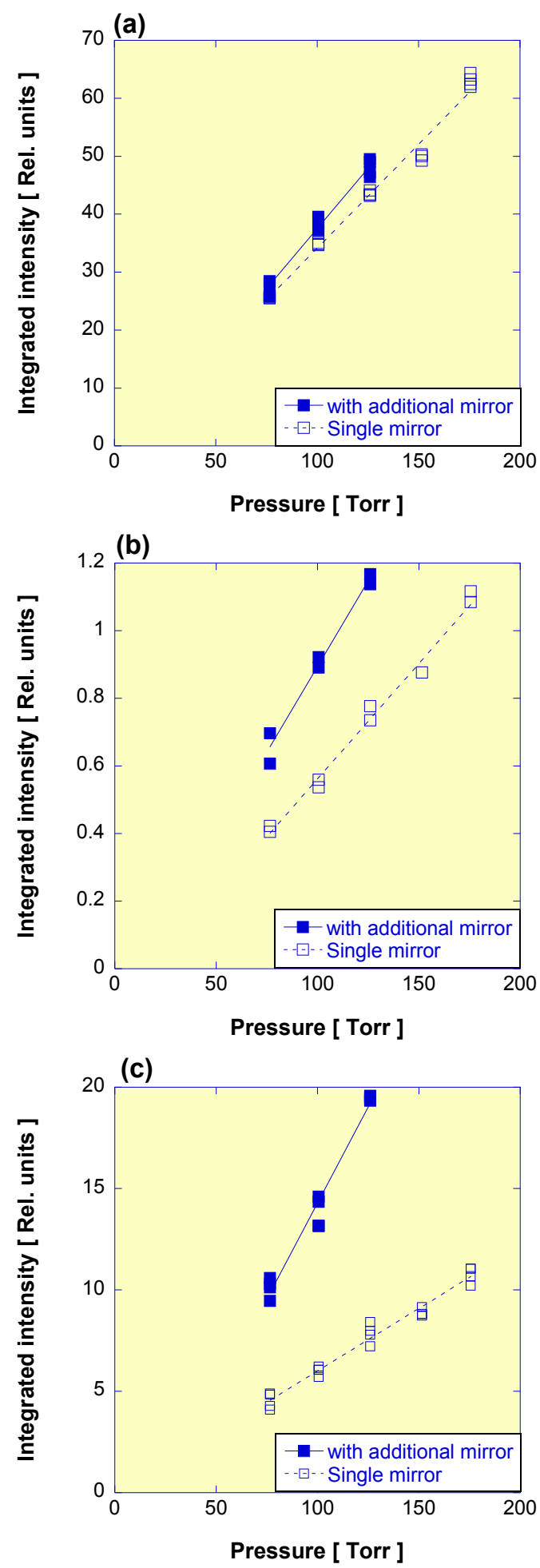

Figure. 2 Integrated intensities with and without additional mirror against nitrogen gas pressures at $\mathrm{X}=-10 \mathrm{~cm}(\mathrm{a}),-15 \mathrm{~cm}$ (b), and $-20 \mathrm{~cm}(\mathrm{c})$.

$-20 \mathrm{~cm}$, we could not obtain the TS signals. The TS signal intensities at $\mathrm{X}=-10 \mathrm{~cm}$ and -15 
$\mathrm{cm}$ with additional mirror are about 1.2 and 1.9 times those without it, respectively. The
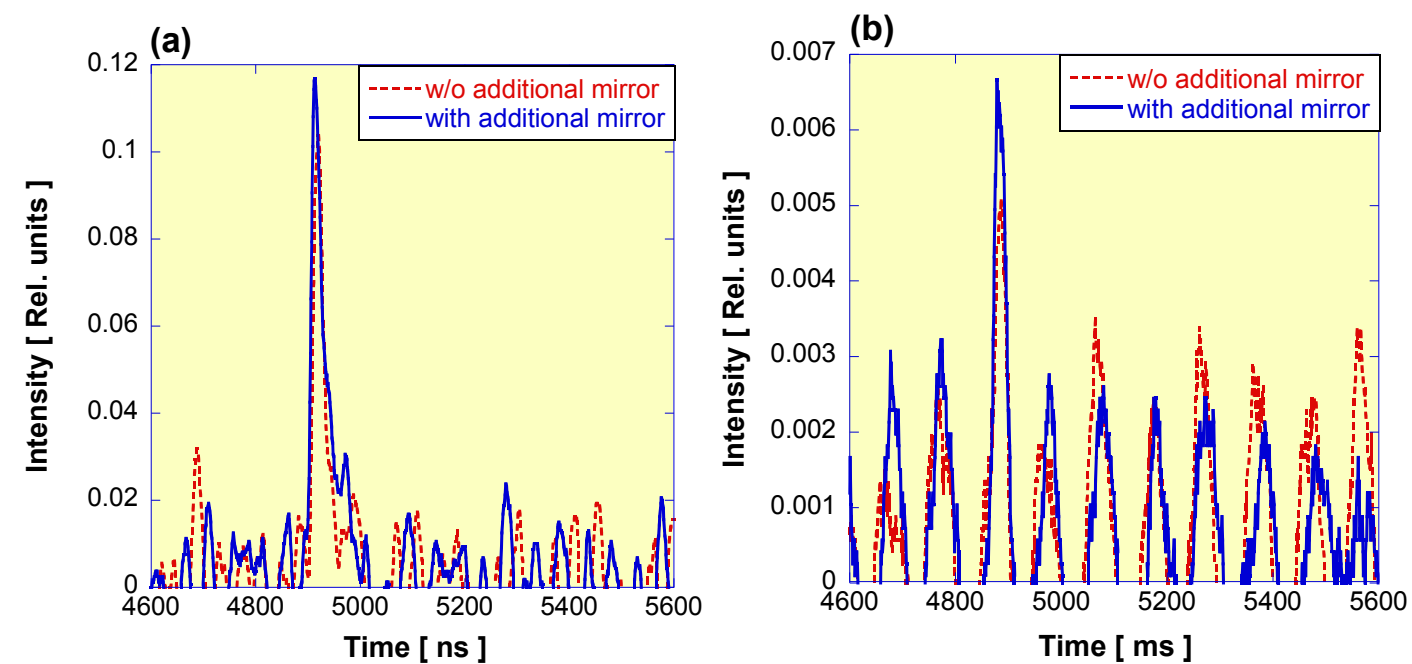

Figure 3. Integrated TS signals with (blue line) and without the additional mirror (red dotted line) at $\mathrm{X}=-10 \mathrm{~cm}$ (a) and $-15 \mathrm{~cm}(\mathrm{~b})$.

intensity increase at $X=-10 \mathrm{~cm}$ is almost the same as the Raman scattering result. The increase at $\mathrm{X}=-15 \mathrm{~cm}$ is different from the Raman scattering result because of the small signal level against the background noise level. The additional mirror is effective to increase the scattering light at the edge plasma region.

\section{Radial electron temperature and density measurements}

We used the TS system to measure radial profiles of electron temperature and density in the GAMMA 10/PDX plasma. The hydrogen plasma is produced from $\mathrm{t}=$ 50 to $240 \mathrm{~ms}$ with heating by RF1 from $\mathrm{t}=$ 51 to $\mathrm{t}=240 \mathrm{~ms}$ and RF2 from $\mathrm{t}=53$ to $\mathrm{t}=$ $240 \mathrm{~ms}$ and east anchor heating by RF3 from $\mathrm{t}=200$ to $240 \mathrm{~ms}$. Figure 4 shows the temporal evolutions of diamagnetism (dotted red line) and electron line density (solid blue line). The YAG laser was injected at $\mathrm{t}=60.0 \mathrm{~ms}$ and $160.0 \mathrm{~ms}$, which are indicated in Fig. 4 in blue lines. In Fig. 5 (a), the red and blue squares show the radial electron temperatures at $\mathrm{t}=60.0 \mathrm{~ms}$ and $160.0 \mathrm{~ms}$, respectively. In Fig. 5 (b), the red and blue squares show the radial

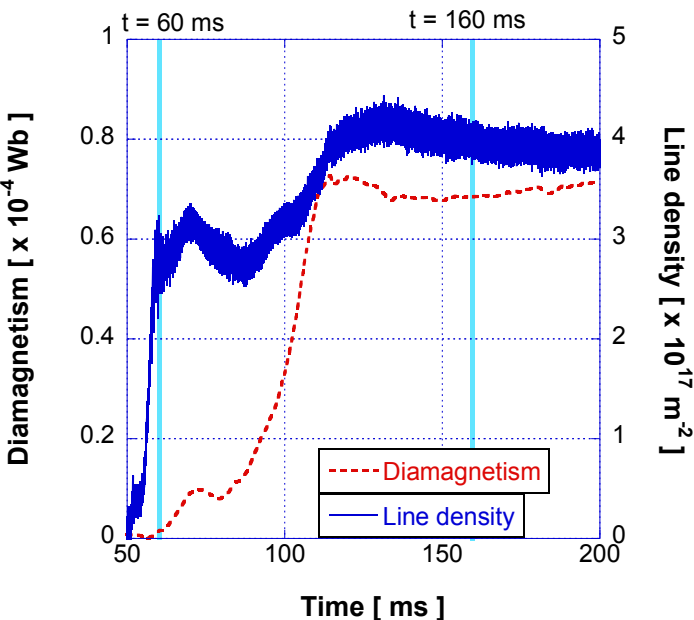

Figure 4. Time evolution of electron line density and diamagnetism. electron densities at $t=60.0 \mathrm{~ms}$ and 160.0 $\mathrm{ms}$, respectively. At the plasma center, the electron temperature and density are about $35 \mathrm{eV}$ and $1.0 \times 10^{18} \mathrm{~m}^{-3}$, respectively, at $\mathrm{t}=60.0 \mathrm{~ms}$ and $57 \mathrm{eV}$ and $1.4 \times 10^{18} \mathrm{~m}^{-3}$, respectively, at $\mathrm{t}=$ $160.0 \mathrm{~ms}$. By changing the YAG laser injection time shot-by-shot, we can obtain the time evolutions of electron temperature and density. In Fig. 6 (a) and (b), the time evolutions of electron temperature and density, respectively, are shown. In Fig. 6 (b), the electron density 
measured using a multi-channel 70-GHz interferometer system [13] is also presented. The timedependent electron density measured using the TS system is almost the same as that measured by the interferometer system. By using the TS system, we could successfully obtain the timedependent electron density and temperature in smaller amounts of plasma shots.
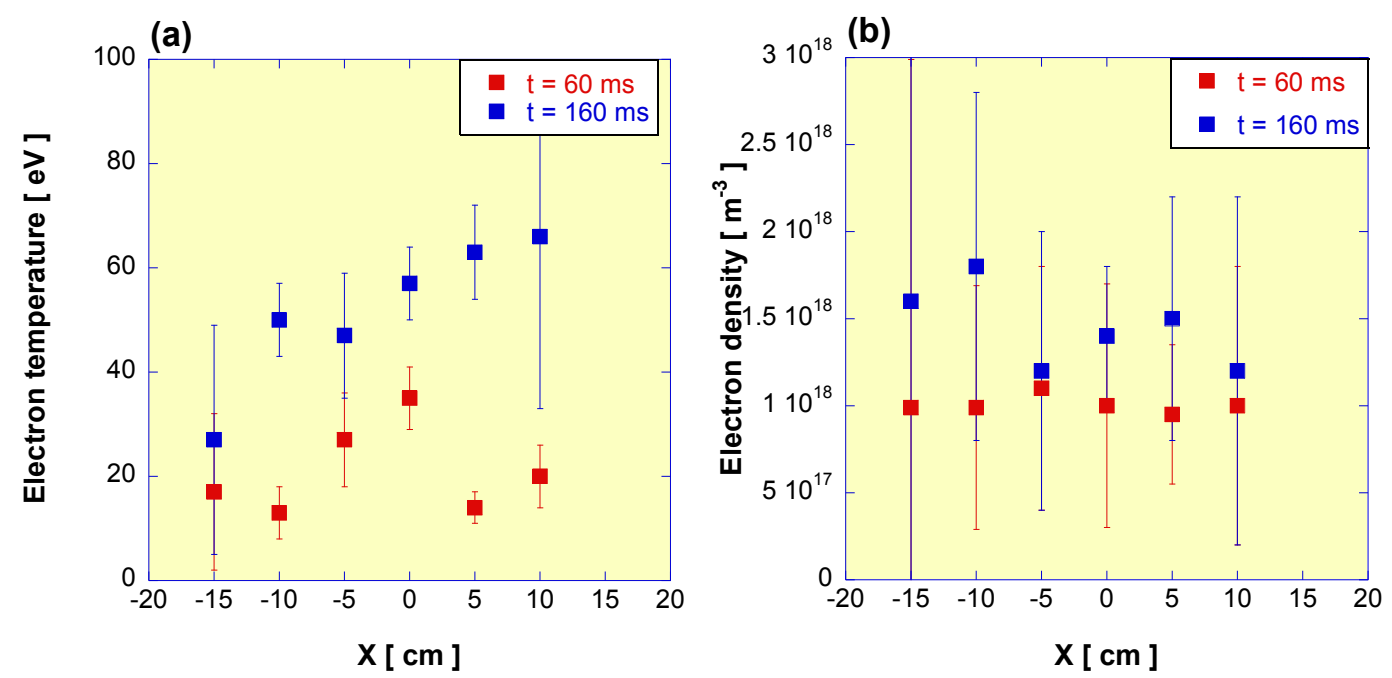

Figure 5. Radial profiles of electron temperature (a) and density (b) at $\mathrm{t}=60.0 \mathrm{~ms}$ and 160.0 ms.
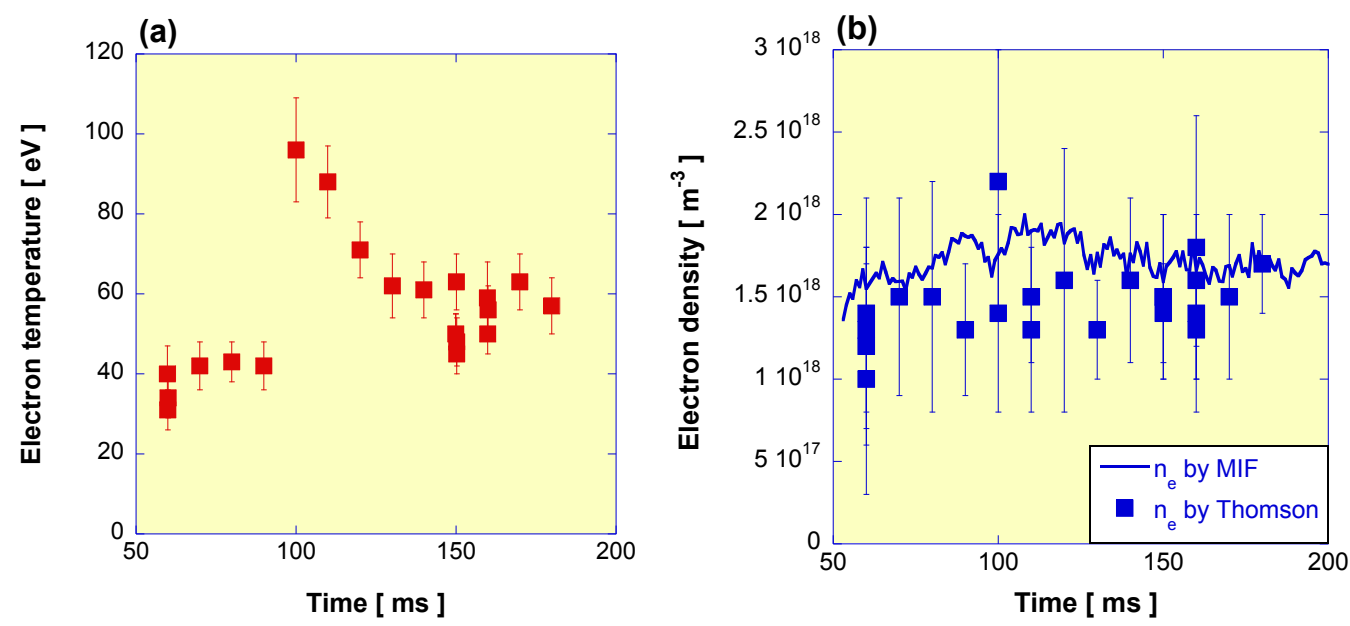

Figure 6. Time evolutions of electron temperature (a) and density (b). In Fig. 6 (b), electron density measured using a multi-channel interferometer system is also shown.

\section{Summary}

We improved the TS system by including the additional mirror to increase the TS signal intensity at the edge plasma region and adding the measuring channels of radial TS signals. The radial electron density and temperature at six positions are successfully obtained. The multitime TS signals are acquired and consequently, the time-dependent radial electron temperatures and densities are measured in smaller amounts of plasma shots. 


\section{Acknowledgments}

The authors thank the members of the GAMMA 10 group of the University of Tsukuba for their collaboration. This study was conducted with the support and under the auspices of the NIFS Collaborative Research Program, NIFS11KUGM056.

\section{References}

[1] K. Narihara et al., Development of Thomson scattering diagnostics for the large helical device, Fusion Eng. Design 34-35, 67 (1997).

[2] K. Narihara, I. Yamada, H. Hayashi and K. Yamauchi, Design and performance of the Thomson scattering diagnostic on LHD, Rev. Sci. Instrum. 72, 1122 (2001).

[3] S. Kainaga et al., Development of a compact Thomson scattering system for the TST-2 spherical Tokamak, Plasma Fusion Res. 3, 027(2008).

[4] H. G. Lee et al., Measurements of the electron temperature by the Thomson scattering system on the Hanbit magnetic mirror device, Rev. Sci. Instrum. 72, 1118 (2001).

[5] A. Mase et al., Laser-Aided Plasma diagnostics on Gamma 10, in Proceedings of Kyushu International Symposium on Laser-Aided Plasma Diagnostics, Nov., 1-3, 1983, Fukuoka, Japan. KIS-LAPD-83/1p-3, 319.

[6] M. Tsalas, et al., Feasibility study for a new high resolution Thomson scattering system for the ASDEX Upgrade pedestal, JINST, 7, C03015 (2012).

[7] T. Hatae, et al., YAG laser Thomson scattering diagnostic on the JT-60U, Rev. Sci. Instrum. 70, 772 (1999).

[8] M. Yoshikawa et al., Measuring electron temperature in the tandem mirror GAMMA 10 plasma using a yttrium-aluminium-garnet Thomson scattering system, Plasma Fusion Res. 6, 1202095 (2011).

[9] M. Yoshikawa et al., Radial electron temperature measurements by using newly installed Thomson scattering system in GAMMA 10, JINST, 7, C03003 (2012).

[10] M. Yoshikawa, et al., Electron temperature and density measurements by using the Thomson scattering system in the tandem mirror GAMMA 10, JINST, 8, C10016 (2013).

[11] M. Yoshikawa, et al., Development of Thomson Scattering System In GAMMA 10, Fusion. Sci. Technol., 68, 99 (2015).

[12] T. Imai, et al., GAMMA 10/PDX Project Status and Future, Trans. Fusion Sci. Technol. 63, 8 (2013).

[13] Y. Nakashima, et al., Recent Results of Divertor Simulation Experiments Using D-module in the GAMMA 10/PDX Tandem Mirror, Trans. Fusion. Sci. Technol. 68, 28 (2014).

[14] Y. Hasegawa, et al., Radial Electron Density Measurement by Using a Multi-Channel Microwave Interferometer in the Tandem Mirror GAMMA 10, Trans. Fusion Sci. Technol. 63, 8 (2013). 